$18^{\text {th }}$ Conference of the Egyptian Society of Animal Production, Hurghada, Egypt, November 7 - 10,2018

\title{
EFFECT OF SYPPLEMENTATION OF MORJORAM AND THYME ON RUMEN FERMENTATION PARAMETERS
}

\author{
H.A.F. Rahmy ${ }^{1}$, H.M. EI Bana ${ }^{1}$, N.E. El-bordeny ${ }^{2}$, Adel E.M. Mahmoud ${ }^{1}$ and Wafaa M.A. Ghoneem ${ }^{1}$ \\ 1-Animal production research department, faculty of Agriculture, Cairo university,12613 Giza, Egypt., 2- Animal \\ Production research Department, Faculty of Agriculture, Ein Shams University, Giza, Egypt
}

The objective of this study was to evaluate the effect of adding morjoram and thyme plants at $4,12,20,28 \mathrm{mg}$ to the dry matter used in the incubation media of an in-vitro rumen model. Digestibility of dry matter (IVDMD), organic matter digestibility (IVOMD), total gas production (TG) , pH , ammonia ( NH3) and Volatile fatty acid (VFA) were studied. Compared to control, $\mathrm{pH}$ was affected with supplementation of plants at different levels, and was significant with thyme at level of $28 \mathrm{mg}$, and with marjoram at level of $20 \mathrm{mg}$.. Significant differences were also observed between lowest level added of both plant, compared to control for $\mathrm{NH}_{3}$, while for VFA and IVDMD, there were no significant difference among the groups. IVOMD was significantly higher in thyme at both levels 20 and $28 \mathrm{mg}$ at $45.6 \%, 46.85 \%$, compared with control at $41.3 \%$, respectively. Total gas (TG) production was significantly higher at level $28 \mathrm{mg}$ in both plants compared with control group.

Based on these results, it may be concluded that addition of medicinal and aromatic herbal plants (marjoram (Origanum majorana), thyme (Thymus vulgaris)), especially at highest levels tested, has a great potential in manipulating rumen fermentation, which may be of benefit when applied in ruminant nutrition.

Keywords: Marjoram, thyme, in vitro, digestibility 